\title{
Identification of the metabolites of ivermectin in humans
}

\author{
Phornpimon Tipthara $^{1}$ () | Kevin C. Kobylinski ${ }^{2}$ () | Markus Godejohann ${ }^{3}$ |

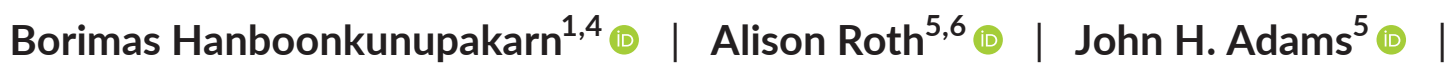 \\ Nicholas J. White $^{1,7} \oplus \mid$ Podjanee Jittamala ${ }^{1,8} \odot \mid$ Nicholas P. J. Day ${ }^{1,7} \oplus$ | Joel Tarning ${ }^{1,7}$
}

${ }^{1}$ Mahidol Oxford Tropical Medicine Research Unit, Faculty of Tropical Medicine, Mahidol University, Bangkok, Thailand

${ }^{2}$ Department of Entomology, Armed Forces Research Institute of Medical Sciences, Bangkok, Thailand

${ }^{3} \mathrm{AlC}$, Bruker BioSpin GmbH, Rheinstetten, Germany

${ }^{4}$ Department of Clinical Tropical Medicine, Faculty of Tropical Medicine, Mahidol University, Bangkok, Thailand

${ }^{5}$ Center for Global Health and Infectious Diseases Research, College of Public Health, University of South Florida, Tampa, FL, USA

${ }^{6}$ Department of Drug Discovery,

Experimental Therapeutics Branch, Walter Reed Army Institute of Research, Silver

Spring, MD, USA

${ }^{7}$ Centre for Tropical Medicine and Global Health, Nuffield Department of Clinical Medicine, University of Oxford, Oxford, United Kingdom

${ }^{8}$ Department of Tropical Hygiene, Faculty of Tropical Medicine, Mahidol University, Bangkok, Thailand

\section{Correspondence}

Joel Tarning, Mahidol Oxford Tropical

Medicine Research Unit, Faculty of Tropical

Medicine, Mahidol University, 420/6

Rajvithi Road, Bangkok 10400, Thailand.

Email: joel@tropmedres.ac

\section{Funding information}

Wellcome Trust, Grant/Award Number: 104926/Z/14/Z: 106698/B/14/Z:

106698/Z/14/Z; Bill and Melinda Gates

Foundation, Grant/Award Number: INV-

006052 and OPP1180249; National Institutes

of Health, Grant/Award Number: R21Al149730

\begin{abstract}
Mass drug administration of ivermectin has been proposed as a possible malaria elimination tool. Ivermectin exhibits a mosquito-lethal effect well beyond its biological half-life, suggesting the presence of active slowly eliminated metabolites. Human liver microsomes, primary human hepatocytes, and whole blood from healthy volunteers given oral ivermectin were used to identify ivermectin metabolites by ultra-high performance liquid chromatography coupled with high-resolution mass spectrometry. The molecular structures of metabolites were determined by mass spectrometry and verified by nuclear magnetic resonance. Pure cytochrome P450 enzyme isoforms were used to elucidate the metabolic pathways. Thirteen different metabolites (M1M13) were identified after incubation of ivermectin with human liver microsomes. Three (M1, M3, and M6) were the major metabolites found in microsomes, hepatocytes, and blood from volunteers after oral ivermectin administration. The chemical structure, defined by LC-MS/MS and NMR, indicated that M1 is 3"-O-demethyl ivermectin, $M 3$ is 4-hydroxymethyl ivermectin, and M6 is 3"-O-demethyl, 4-hydroxymethyl ivermectin. Metabolic pathway evaluations with characterized cytochrome P450 enzymes showed that M1, M3, and M6 were produced primarily by CYP3A4, and that M1 was also produced to a small extent by CYP3A5. Demethylated (M1) and hydroxylated (M3) ivermectin were the main human in vivo metabolites. Further studies are needed to characterize the pharmacokinetic properties and mosquito-lethal activity of these metabolites.
\end{abstract}

KEYWORDS

ivermectin, LC-MS/MS, malaria, metabolism

Abbreviations: CYP450, cytochrome P450; HLM, human liver microsomes; HMBC, heteronuclear multiple bond correlation; HRMS, high-resolution mass spectrometry; HSQC, heteronuclear single quantum correlation; IVM, ivermectin; MDA, mass drug administration; NADPH, reduced form of nicotinamide adenine dinucleotide phosphate; NMR, nuclear magnetic resonance; NTDs, neglected tropical diseases; PHH, primary human hepatocytes; UHPLC-Q-TOF-MS, ultra-high performance liquid chromatography quadrupole time-of-flight mass spectrometry.

This is an open access article under the terms of the Creative Commons Attribution License, which permits use, distribution and reproduction in any medium, provided the original work is properly cited.

(C) 2021 The Authors. Pharmacology Research \& Perspectives published by John Wiley \& Sons Ltd, British Pharmacological Society and American Society for Pharmacology and Experimental Therapeutics. 


\section{1 | INTRODUCTION}

Ivermectin (IVM) is an antiparasitic and endectocidic drug used for decades in animal health and for treating onchocerciasis, lymphatic filariasis, scabies, and strongyloidiasis in humans. ${ }^{1}$ IVM also has some antiviral activity including against SARS-CoV-2 in vitro. ${ }^{2}$

Malaria is a mosquito-borne disease transmitted by Anopheles mosquitoes during blood feeding. Numerous studies have reported the mosquito-lethal effect of $\mathrm{IVM}^{3-5}$ and the ability to inhibit sporogony of Plasmodium in the mosquito. ${ }^{6-8}$ Mass drug administration (MDA) of IVM has been suggested as a possible vector control tool to aid malaria elimination as it has been shown to reduce Plasmodium transmission by mosquitoes ${ }^{9}$ and reduce transmission to humans. ${ }^{10}$ A recent clinical trial in Thailand showed that mosquito-lethal effects persisted well beyond the detectable presence of the parent compound, which suggests that IVM may have active metabolites that are more slowly eliminated than the parent compound. ${ }^{5}$

IVM is a semisynthetic compound derived from avermectin ( $B_{1}$ series), a natural fermentation product of the soil bacterium Streptomyces avermitilis. The regiospecific hydrogenation of avermectin $B_{1}$ at the 22,23-double bond produces the 22,23-single bond derivative called 22,23-dihydroavermectin $\mathrm{B}_{1}$ or IVM. ${ }^{11}$ IVM is a mixture containing at least $90 \%$ of 22,23 -dihydroavermectin $B_{1}$ $\left(\mathrm{H}_{2} \mathrm{~B}_{1 \mathrm{a}}\right.$ or IVM- $\left.\mathrm{B}_{1 \mathrm{a}}\right)$ and less than $10 \%$ of 22,23 -dihydroavermectin $\mathrm{B}_{1 \mathrm{~b}}\left(\mathrm{H}_{2} \mathrm{~B}_{1 \mathrm{~b}}\right.$ or IVM- $\left.\mathrm{B}_{1 \mathrm{~b}}\right)$. The chemical structures show an alkyl side chain difference between IVM- $B_{1 a}$ and IVM- $B_{1 b}$ at C25 (Figure 1). Both compounds have the same antiparasitic activity. ${ }^{12}$

There have been several studies of IVM metabolites produced in nonhuman vertebrates. ${ }^{13-17}$ The major metabolite found in rats, cattle, and sheep is the 24-hydroxymethyl derivative ${ }^{13,16}$ while only trace levels are found in pigs. The 3 "-O-demethyl derivative is the major metabolite present in pigs. ${ }^{14} \mathrm{~A}$ previous in vitro study using human liver microsomes found nine IVM metabolites, mostly hydroxylated and demethylated compounds including the two listed above. ${ }^{18}$

In this study, we aimed to identify the common metabolites in humans through in vitro and in vivo experiments. Pooled human

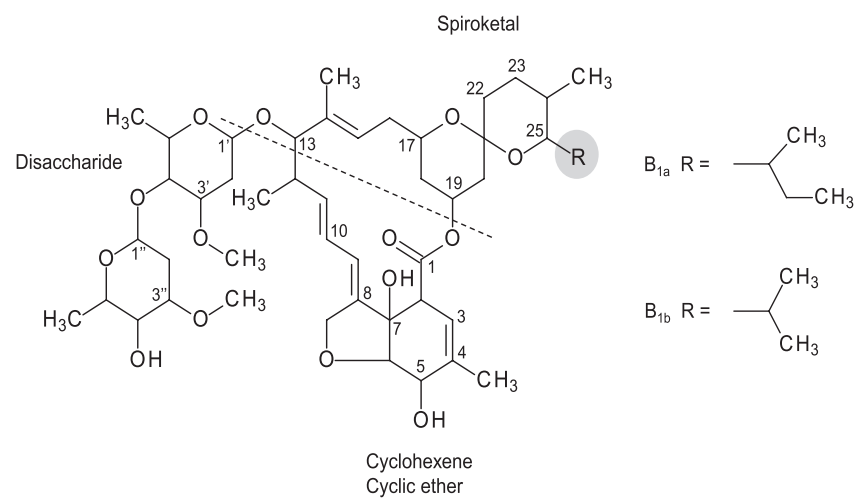

FIGURE 1 Molecular structure of ivermectin. IVM consists of a spiroketal unit (C17-C28), cyclohexene cyclic ether unit (C2-C8), and a disaccharide unit at C13. A secondary butyl side chain at C25 give rise to the major component (IVM- $\left.\mathrm{B}_{1 \mathrm{a}}\right)$ and an isopropyl side chain give rise to the minor component $\left(<10 \%\right.$; IVM- $\left.B_{1 b}\right)$ liver microsomes and primary human hepatocytes were exposed to IVM, and metabolite fractions were collected to identify metabolites produced in vitro. Human whole blood, collected from healthy volunteers after a single oral dose of IVM $(400 \mu \mathrm{g} / \mathrm{kg})$, was used to identify metabolites produced in vivo. While the standard dose of ivermectin is $200 \mu \mathrm{g} / \mathrm{kg}$, the $400 \mu \mathrm{g} / \mathrm{kg}$ dose has been shown to be safe and efficacious in numerous clinical trials, is frequently used in clinical settings, and is now recommended on the package insert for lymphatic filariasis mass drug administration. Previous pharmacokinetic modeling and in vitro mosquito mortality experiments indicated that IVM $400 \mu \mathrm{g} / \mathrm{kg}$ is the ideal dose to target the important Anopheles malaria vectors in the Greater Mekong Subregion. ${ }^{8}$ The structure of IVM metabolites were characterized by LC-MS/MS and verified by NMR. The metabolic pathways that generated these metabolites were characterized by incubation of IVM with purified human cytochrome P450 (CYP) enzymes, followed by LC-MS/MS analysis.

\section{2 | MATERIALS AND METHODS}

\section{1 | Chemicals}

IVM (IVM- $\left.B_{1 a}>95 \%, I V M-B_{1 b}<2 \%\right)$ was purchased from SigmaAldrich. IVM-B Ia (95\% purity) was purchased from Toronto Research Chemicals Inc. IVM-B ${ }_{1 \mathrm{~b}}(99.27 \%$ purity) was purchased from Clearsynth Labs Ltd. Pooled human liver microsomes (50 donors, Gibco $^{\mathrm{TM}}$ ) were purchased from Thermo Fisher Scientific. Cryopreserved primary human hepatocytes (M00995-P, UBV donor) and hepatocyte culture medium (InVitroGRO ${ }^{\mathrm{TM}} \mathrm{CP}$ Medium) were purchased from BiolVT LLC. cDNA-expressed human CYP isoenzymes $1 \mathrm{~A} 2,2 \mathrm{~B} 6,2 \mathrm{C} 8,2 \mathrm{C} 9,2 \mathrm{C} 18,2 \mathrm{C} 19,2 \mathrm{D} 6,2 \mathrm{E} 1,3 \mathrm{~A} 4$, and 3A5 (Corning ${ }^{\circledR}$ Supersomes ${ }^{\mathrm{TM}}$ ), insect cell control microsomes, human P450 cytochrome $b_{5}$ oxidoreductase, and NADPH regenerating system were purchased from Corning Inc. LC-MS grade water and acetonitrile were purchased from J.T Baker. LC-MS grade ammonium acetate, formic acid, and HPLC grade organic solvent for NMR analysis were purchased from Sigma-Aldrich. $\mathrm{CD}_{3} \mathrm{CN} 99.8 \%$ and DCOOD $98 \%$ were purchased from Deutero $\mathrm{GmbH}$. Water for NMR was prepared from a Milli-Q purification system from Merck.

\section{2 | Pooled human liver microsomes}

Pooled human liver microsomes (containing $20 \mathrm{mg} / \mathrm{ml}$ of protein) were thawed on ice. Microsome reactions were performed in microcentrifuge tubes by adding $183 \mu \mathrm{l}$ of $0.1 \mathrm{M}$ potassium phosphate buffer ( $\mathrm{pH}$ 7.4), $2 \mu \mathrm{l}$ of $1.0 \mathrm{mM}$ IVM (prepared in acetonitrile $80 \%$ $(\mathrm{v} / \mathrm{v}))$, and $5 \mu \mathrm{l}$ of microsomes. The tube was vortexed briefly and incubated at $37^{\circ} \mathrm{C}$ for $5 \mathrm{~min}$ in a shaking water bath. The reaction was initiated by adding $10 \mu \mathrm{l}$ of $20 \mathrm{mM} \mathrm{NADPH}$ (reduced form of nicotinamide adenine dinucleotide phosphate) prepared in $100 \mathrm{mM}$ potassium phosphate buffer $\mathrm{pH}$ 7.4. Total reaction volume per tube 
was $200 \mu \mathrm{l}$ with the final concentration of $10 \mu \mathrm{M}$ IVM and $1.0 \mathrm{mM}$ $\mathrm{NADPH}$. Each tube was vortexed briefly and a baseline sample (0 min control) was collected by aliquoting $100 \mu \mathrm{l}$ of the metabolite fraction mixture described above to a separate tube with $100 \mu \mathrm{l}$ of pre-chilled acetonitrile, which was kept on ice until centrifugation. Two separate negative control tubes were prepared, one without NADPH (negative co-factor control) but with an extra $10 \mu \mathrm{l}$ of buffer and a second tube without IVM substrate (negative IVM control) with an extra $2 \mu \mathrm{l}$ of buffer. All remaining reactions, including negative co-factor control, and negative IVM control, were incubated at $37^{\circ} \mathrm{C}$ for $60 \mathrm{~min}$ with gentle shaking. After 60 min of incubation, all tubes were removed from the water bath and cold acetonitrile was added immediately to make a final 1:1 (v/v) ratio. All tubes (0- and 60-min reactions, negative co-factor, and negative IVM controls) were vortexed briefly again and centrifuged at $10,000 \mathrm{~g}$ for $15 \mathrm{~min}$ at $4^{\circ} \mathrm{C}$. The supernatant was collected and kept frozen at $-80^{\circ} \mathrm{C}$ until LC-MS/MS analysis. The reactions described above were performed using IVM, pure IVM- $B_{1 a}$ and pure IVM- $\mathrm{B}_{1 \mathrm{~b}}$ as substrates (biological triplicate incubations for each substrate).

For NMR analysis, 60 tubes were prepared each with $10 \mu \mathrm{l}$ of $1.0 \mathrm{mM}$ IVM, $915 \mu \mathrm{l}$ of $100 \mathrm{mM}$ potassium phosphate buffer $(\mathrm{pH}$ 7.4), $25 \mu \mathrm{l}$ of microsomes, and $50 \mu \mathrm{l}$ of $20 \mathrm{mM} \mathrm{NADPH}$ for a final volume of $1 \mathrm{ml}$. Reactions were stopped at $60 \mathrm{~min}$ with ice-cold acetonitrile and centrifuged immediately as describe above. The supernatant was collected, evaporated in speed vacuum, and kept frozen at $-80^{\circ} \mathrm{C}$ until NMR analysis.

\section{3 | Primary human hepatocytes}

Primary human hepatocytes were seeded on a 384-well plate as described previously to stimulate reacquisition of in vivo physiologic activity. ${ }^{19}$ At day 3 post seed, IVM $(10 \mu \mathrm{M})$ was added to each well. There were no media changes and no subsequent additions of IVM. Forty microliters of media was collected from five individual wells at 24 hours and pooled in a microcentrifuge tube (total volume $200 \mu \mathrm{l}$ ). Forty microliters of media was mixed with cold acetonitrile $(160 \mu \mathrm{l})$, vortexed for $10 \mathrm{~min}$ at ambient temperature, and centrifuged at $10,000 \mathrm{~g}$ for $15 \mathrm{~min}$ at $4^{\circ} \mathrm{C}$ (triplicate extractions). The supernatant was collected, evaporated in speed vacuum, and kept frozen at $-80^{\circ} \mathrm{C}$ until LC-MS/MS analysis.

\subsection{Healthy volunteer samples}

Venous blood was collected from three healthy Thai volunteers given a single oral dose of IVM $(400 \mu \mathrm{g} / \mathrm{kg})\left(\right.$ NCT02568098). ${ }^{5}$ The blood samples were collected in sodium heparin tubes at $24 \mathrm{~h}$ postIVM ingestion and kept frozen at $-80^{\circ} \mathrm{C}$ until LC-MS/MS analysis. For metabolite extraction, whole blood samples were thawed at ambient temperature, vortexed briefly, and centrifuged at $3000 \mathrm{~g}$ for $5 \mathrm{~min}$ at $20^{\circ} \mathrm{C}$. Fifty microliters of plasma was transferred into a new microcentrifuge tube and mixed with $200 \mu \mathrm{l}$ of cold acetonitrile.
Tubes were vortexed for $10 \mathrm{~min}$ at ambient temperature and centrifuged at $1100 \mathrm{~g}$ for $5 \mathrm{~min}$ at $20^{\circ} \mathrm{C}$. The supernatant was collected, evaporated in speed vacuum, and kept at $-80^{\circ} \mathrm{C}$ freezer until LCMS/MS analysis.

\section{5 | cDNA-expressed cytochrome P450 enzyme}

The metabolism of IVM was studied in vitro using cDNA-expressed human CYP isoenzymes 1A2, 2B6, 2C8, 2C9, 2C18, 2C19, 2D6, 2E1, 3A4, 3A5 (1.0 $\mu \mathrm{M})$. In separate tubes for each CYP isoform, the following reagents were added: $166 \mu \mathrm{l}$ of $0.1 \mathrm{M}$ potassium phosphate buffer ( $\mathrm{pH}$ 7.4), $2 \mu \mathrm{l}$ of $1.0 \mathrm{mM} \mathrm{IVM} \mathrm{(prepared} \mathrm{in} \mathrm{acetonitrile} 80 \%$ $(\mathrm{v} / \mathrm{v}))$, and $20 \mu \mathrm{l}$ of CYP enzyme. A separate tube was prepared for the negative control (no human CYP enzyme) by adding $20 \mu \mathrm{l}$ of insect cell control microsomes. A second tube of negative control was prepared, to evaluate the effect of reductase, by adding $20 \mu \mathrm{l}$ of human P450 cytochrome $b_{5}$ oxidoreductase. The tubes were vortexed briefly and incubated at $37^{\circ} \mathrm{C}$ for $5 \mathrm{~min}$ in a shaking water bath. The reactions were initiated by adding $12 \mu \mathrm{l}$ of NADPH regenerating system solution (premixed $10 \mu \mathrm{l}$ of solution $A$ and $2 \mu \mathrm{l}$ of solution $B$ ). Each tube was vortexed briefly and a baseline sample (0 min control) was collected by aliquoting $100 \mu$ of the incubation to a separate tube with $100 \mu \mathrm{l}$ of prechilled acetonitrile, which was held on ice until centrifugation. All tubes (reactions and controls) were incubated at $37^{\circ} \mathrm{C}$ for $60 \mathrm{~min}$ with gentle shaking. After $60 \mathrm{~min}$ of incubation, all tubes were removed from the water bath and cold acetonitrile was added immediately at a 1:1 ratio $(\mathrm{v} / \mathrm{v})$. Tubes were vortexed briefly and centrifuged at $10,000 \mathrm{~g}$ for $15 \mathrm{~min}$ at $4^{\circ} \mathrm{C}$. The clear supernatant was collected into new microcentrifuge tubes and kept frozen at $-80^{\circ} \mathrm{C}$ until LC-MS/MS analysis. The incubations and reactions described above were performed in triplicate.

\section{6 | UHPLC-Q-TOF-MS}

The LC-MS/MS system used was an ultra-high performance liquid chromatography (Agilent 1260 Quaternary pump, Agilent 1260 High Performance autosampler, and Agilent 1290 Thermostatted Column Compartment SL, Agilent Technologies) coupled to a quadrupole time-of-flight mass spectrometer (Q-TOF-MS) (TripleTOF $5600^{+}$, Sciex) with an electrospray ionization (ESI) using a DuoSpray ion source. The mobile phase system for UHPLC was water containing $10 \mathrm{mM}$ ammonium acetate and $0.1 \%$ formic acid (mobile phase A) and acetonitrile:water at a 95:5 ratio (v/v) containing $10 \mathrm{mM}$ ammonium acetate and $0.1 \%$ formic acid (mobile phase B). Human liver microsome extracts were transferred directly to a LC vial for injection. Evaporated supernatant from primary human hepatocytes and whole blood samples were reconstituted in 20 and $50 \mu$, respectively, of mobile phase at starting gradient (mobile phase $A: B$ at a ratio of $60: 40(v / v))$ and transferred to a $L C$ vial for injection. $\mathrm{LC}$ vials were kept in the autosampler at $6^{\circ} \mathrm{C}$ during analysis. Five microliters of sample was injected on to a C18 reversed-phase 
column (ACQUITY UPLC HSS T3, $2.1 \times 100$ mm, $1.8 \mu \mathrm{m}$; Waters Corporation) protected by a precolumn (ACQUITY UPLC HSS T3, $2.1 \times 5 \mathrm{~mm}, 1.8 \mu \mathrm{m}$, Waters), for separation by UHPLC at a flow rate of $0.3 \mathrm{ml} / \mathrm{min}$ at $40^{\circ} \mathrm{C}$. The UHPLC elution gradient was started at $40 \%$ mobile phase $B$ for $2.0 \mathrm{~min}$ (0-2.0 min), followed by $40 \%-80 \%$ B for $2.0 \mathrm{~min}$ (2.0-4.0 $\mathrm{min}$ ), 80\%-100\% B for $5.0 \mathrm{~min}$ (4.0-9.0 min), $100 \%$ B for $5.0 \mathrm{~min}(9.0-14.0 \mathrm{~min}), 100 \%-40 \%$ B for $0.1 \mathrm{~min}$ (14.0-14.1), and 40\% B for $3.9 \mathrm{~min}$ (14.1-18.0 min). The UHPLC-QTOF-MS system, mass ion chromatogram, and mass spectra were acquired by Analyst ${ }^{\text {TM }}$ Software version 1.7 (SCIEX). The Q-TOF-MS was operated in ESI-positive mode at ion source gas 1 (GS1) of $40 \mathrm{psi}$, ion source gas 2 (GS2) of $40 \mathrm{psi}$, curtain gas (CUR) of $30 \mathrm{psi}$, ion spray voltage floating (ISVF) of $4500 \mathrm{~V}$, source temperature (TEM) at $350^{\circ} \mathrm{C}$, and declustering potential (DP) of $120 \mathrm{~V}$. Data were acquired in the informative-dependent acquisition (IDA) mode composed of a TOF-MS scan and 10 dependent product ion scans in the high sensitivity mode with dynamic background subtraction. Mass range of TOF-MS scan was at $\mathrm{m} / \mathrm{z} 100-1000$ and product ion scan was at $\mathrm{m} / \mathrm{z}$ 50-1000. IVM standard solution (100 $\mathrm{ng} / \mathrm{ml})$ was injected before and after batch analysis for validating the system performance.

\section{7 | LC-SPE-NMR/MS}

The analyses were performed by in-line instruments of interfacing liquid chromatography with parallel NMR and mass spectrometry. The 60 dried microsome pellets described above were each reconstituted in $300 \mu \mathrm{l}$ of methanol and sonicated for $5 \mathrm{~min}$. Supernatants were pooled into one $50 \mathrm{ml}$ falcon tube. A second extraction of the microsome residue was performed by adding an additional $300 \mu$ l of acetonitrile followed by sonication for $5 \mathrm{~min}$. The supernatant from the second extraction was then transferred to the same $50 \mathrm{ml}$ falcon tube described above. The pooled supernatant was evaporated under nitrogen gas to a final volume of $200 \mu \mathrm{l}$ and transferred to an HPLC vial. The extract (33 $\mu \mathrm{l})$ was injected into the HPLC (Agilent 1260) at a flow rate of $0.5 \mathrm{ml} /$ min at $25^{\circ} \mathrm{C}$ on a $250 \times 4.6 \mathrm{~mm}, 5 \mu \mathrm{m}$ Kinetex EVO C18 column (Phenomenex). Mobile phase A consisted of water with $0.1 \%$ formic acid- $d_{2}$ (DCOOD) and mobile phase $B$ consisted of acetonitrile with $0.1 \%$ DCOOD. The elution gradient was started at $50 \%$ mobile phase $B$ for $2.0 \mathrm{~min}$ (0-2.0 min), followed by a linear increase from $50 \%$ B to $100 \%$ B over the next $33.0 \mathrm{~min}$ (2.0-35.0 min), ending on $100 \%$ B for $5.0 \mathrm{~min}$ (35.0-40.0 min). UV detection was done at $240 \mathrm{~nm}$. An MS Bridge interface (Bruker Biospin) was used to split a small portion of the effluent from the HPLC column and direct it to the ion source of a MicrOTOF-QII mass spectrometer (Bruker Daltonik, Bremen, Germany) using an acetonitrile makeup flow of $70 \mu \mathrm{l} / \mathrm{min}$. The mass spectrometer was operated in the positive ionization mode with a scan range at $\mathrm{m} / \mathrm{z} 50$ to 1000 . Mass calibration was done with sodium acetate infused at the beginning of the chromatography. The isolated metabolites were trapped postcolumn on $2 \times 10 \mathrm{~mm}$ solid phase extraction cartridges filled with HySphere GP resin using the Prospekt 2 SPE interface from Spark Holland. The peaks of three injections (each $33 \mu \mathrm{l}$ ) were combined on individual cartridges (multi trapping). In total, $2 \times 3$ trappings were performed. The makeup flow rate for the trapping was $1.5 \mathrm{ml} / \mathrm{min}$. After chromatography the cartridges including the metabolites were dried with nitrogen gas and the two cartridges containing the metabolites were eluted with each $300 \mu \mathrm{l}$ of acetonitrile- $\mathrm{d}_{3}\left(\mathrm{CD}_{3} \mathrm{CN}\right)$ into $5-\mathrm{mm}$ NMR tubes. In total, six trappings were finally transferred to the NMR tube for each metabolite resulting in a total volume of $600 \mu \mathrm{l}$. The API reference sample was measured with a $500 \mathrm{MHz}$ AVANCE III NMR spectrometer equipped with a nitrogen cooled 5-mm Prodigy $\mathrm{TCl}$ (triple resonance inverse configuration of the coils with a cooled carbon channel) cryoprobe. Postcolumn SPE fractions of the metabolites were measured first with the $500 \mathrm{MHz}$ spectrometer and later with an $800 \mathrm{MHz}$ Neo NMR spectrometer equipped with a helium cooled 5-mm TCl cryoprobe (Bruker Biospin). SPE fractions were analyzed with the CMC-se software using optimized parameter sets including the Proton-1D, edited heteronuclear single coherence spectroscopy (HSQC) and heteronuclear multiple bond coherence spectroscopy (HMBC). In addition, selective HMBC experiments were performed for areas of closely resonating carbon resonances. IVM (5.2 $\mathrm{mg}$ ) was dissolved in deuterated acetonitrile $(1 \mathrm{ml})$ and transferred to a $5-\mathrm{mm}$ NMR tube and run as a reference compound.

\section{8 | Data analysis}

Metabolite identification was done by MetabolitePilot ${ }^{\mathrm{TM}}$ Software version 2.0 (SCIEX). The MS/MS spectrum of IVM was exported as text files by the PeakView software (SCIEX) and imported to MetabolitePilot ${ }^{\mathrm{TM}}$ software (SCIEX) as a reference spectrum for creating the IVM library. Raw data files (.wiff) of metabolite sample analyses were imported to MetabolitePilot ${ }^{\mathrm{TM}}$ software and compared against the IVM-library peak finding strategies as described in the supplementary material (Supplement Appendix S1). For the LC-SPE-NMR/MS system, the HPLC was operated by Hystar 3.2 (Bruker Daltonics), mass spectrum acquired by Microtof control (Bruker Daltonics), and the NMR spectrometer was operated by Topspin 3.5 (Bruker Biospin). The Complete Molecular Confidence - Structure Elucidation (CMC-se) software version 2.6.1 (Bruker Biospin) was used for structure elucidation.

\section{3 | RESULTS}

\section{1 | In vitro and in vivo metabolite identification by UHPLC-Q-TOF-MS}

Thirteen IVM metabolites (M1-M13) were identified from incubations with pooled human liver microsomes as shown in the metabolite chromatogram in Figure 2A. All metabolites were more polar 
(A)

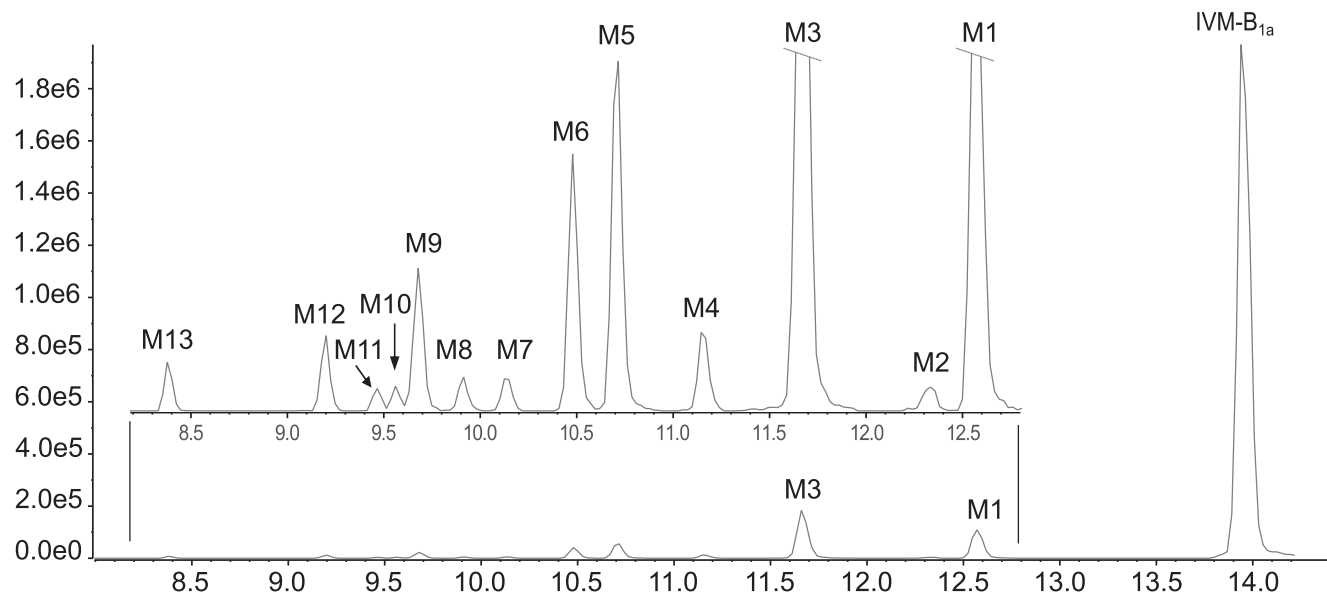

\section{Microsomes}

(B)

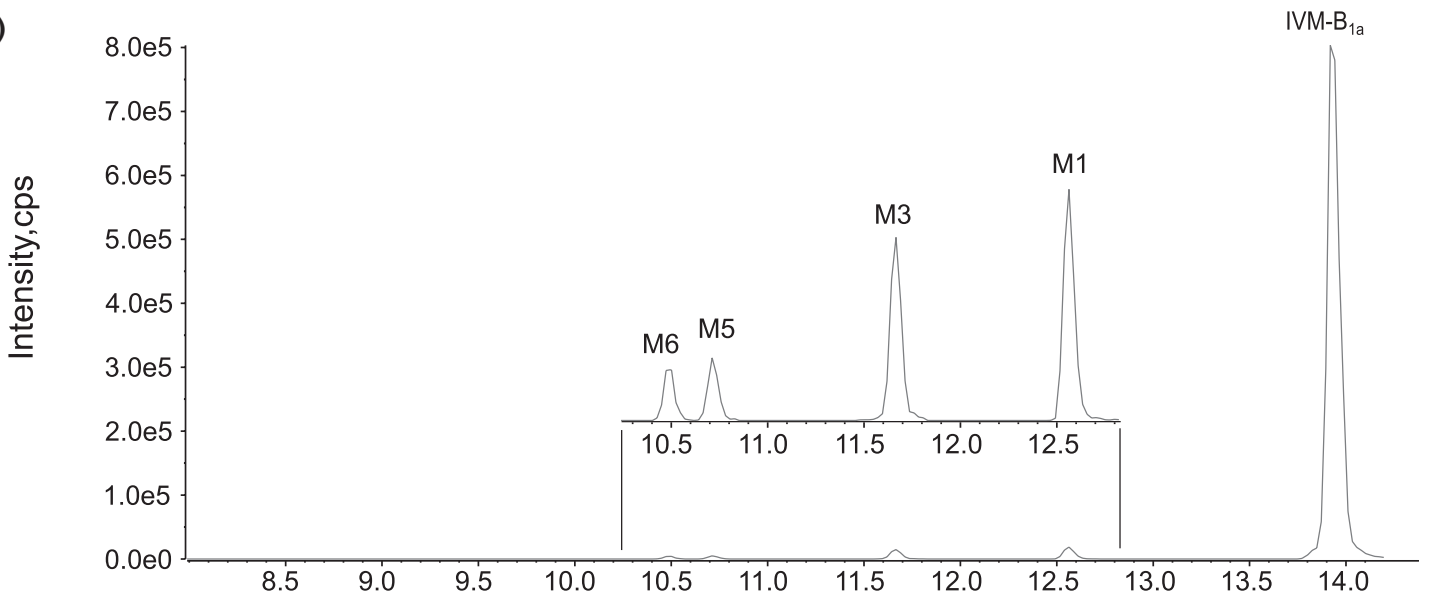

Hepatocytes

(C)

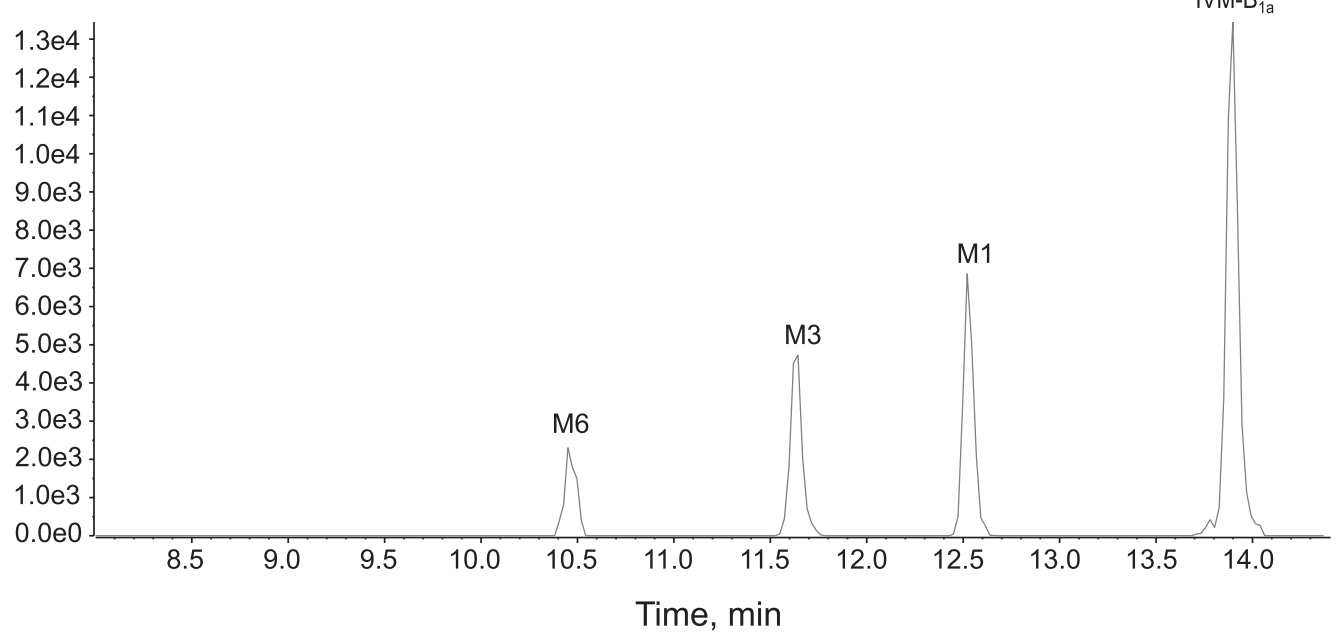

Volunteer Blood

FIGURE 2 Metabolite chromatograms. Chromatogram (A) shows the metabolites identified using pooled human liver microsomes incubated with IVM for $60 \mathrm{~min}$. The upper insert chromatogram shows a zoom of RT 8.2-12.8 min. Chromatogram (B) shows the metabolites identified in the media fraction of primary human hepatocytes exposed to IVM for 24 hours. The upper insert chromatogram shows a zoom of RT 10.3-12.8 min. Chromatogram (C) shows the metabolites identified in human volunteer blood 24 hours after IVM administration

than the IVM parent compound. Four of these metabolites (i.e., M1, M3, M5, and M6) were found also in the medium of IVM-exposed primary human hepatocytes (Figure 2B). The three most abundant metabolites found in microsome and hepatocytes incubations (i.e., M1, M3, and M6) were found also in healthy volunteer blood 24 hours after IVM administration (Figure $2 \mathrm{C}$ ). Reference MS/MS spectra of IVM- $B_{1 a}$ and IVM- $B_{1 b}$ used in the library search were generated from analysis of $100 \mathrm{ng} / \mathrm{ml}$ of IVM standard solution. IVM- $B_{1 \mathrm{~b}}$ $\left(\mathrm{m} / \mathrm{z}\right.$ 878.5) eluted 0.9 min earlier than IVM- $\mathrm{B}_{1 \mathrm{a}}(\mathrm{m} / \mathrm{z}$ 892.5) as shown in the extract ion chromatogram (Figure $3 \mathrm{~A}$ ). The major product ions 
(A)

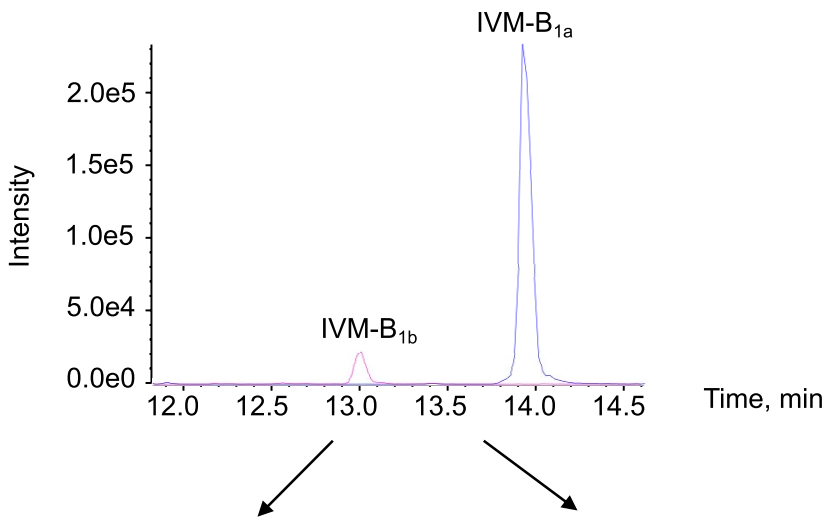

(B)

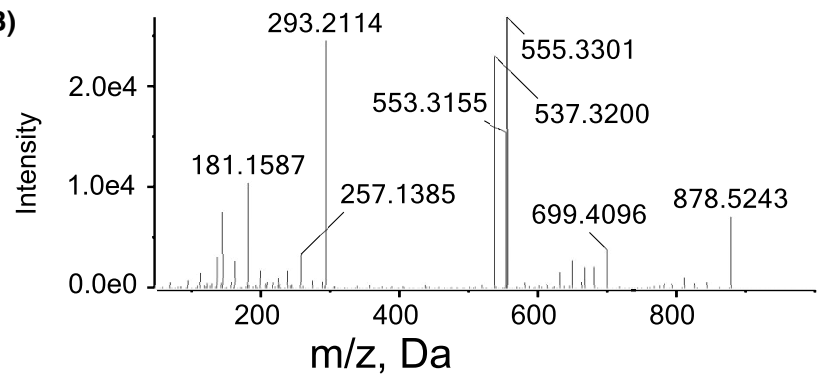

(C)

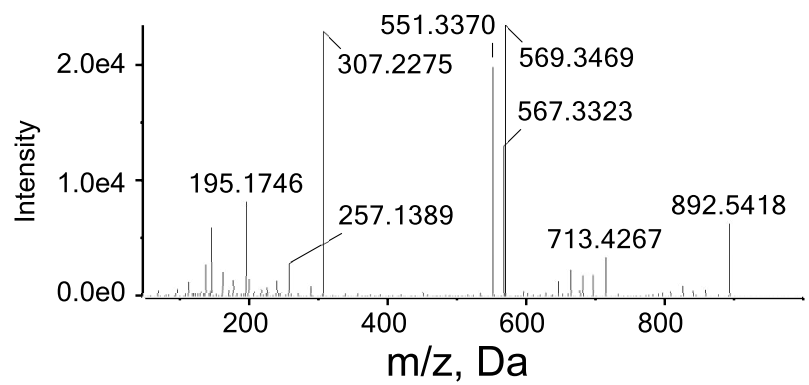

(D)

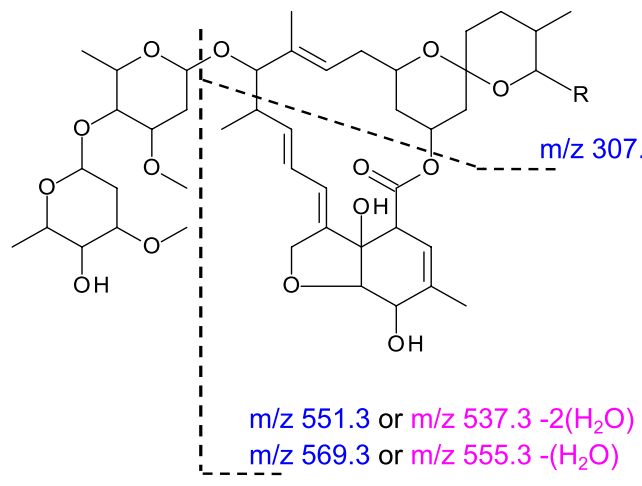

FIGURE 3 Extracted ion chromatogram and MS/MS spectra. The figure shows (A) extracted ion chromatogram of IVM- $B_{1 \mathrm{a}}$ and IVM- $B_{1 \mathrm{~b}}$, (B) MS/MS spectrum of IVM- $B_{1 b}$, (C) MS/MS spectrum of IVM- $B_{1 a}$, and (D) major fragments of IVM

of IVM- $B_{1 b}$ were $m / z$ 293.2, 537.3, and 555.3 (Figure 3B) and those of IVM- $B_{1 a}$ were $m / z$ 307.2, 551.3, and 569.3 (Figure 3C). The fragmentation pattern of the ammonium adduct ion of $I V M-B_{1 a}$ and $I V M-B_{1 b}$ is presented in Figure 3D. The MS/MS spectra of M1 to M13 (Figure 4) were used to define the molecular structure of the metabolites.

\section{2 | Metabolite interpretation by LC-MS/MS}

The thirteen metabolites (M1 to M13) identified from the MetabolitePilot $^{\mathrm{TM}}$ software (Sciex) are reported in Table 1. The molecular ions that were detected in negative controls (including 0 -min incubations, negative co-factor controls, and negative IVM controls) were excluded from the list. Percentage scores were based on three parameters including mass accuracy, mass defect, ${ }^{20,21}$ and MS/MS spectrum (for details see supplementary material). Chemical structures and biotransformation sites of the metabolites (M1 to M13) were interpreted based on the two fragment ions of IVM$B_{1 a}\left(m / z \quad 307.2\right.$ and 551.3; Figure 3). IVM- $B_{1 a}$ metabolites without ion $\mathrm{m} / \mathrm{z} 307.2$ suggested that biotransformation occurred in the spiroketal moiety. The presence of ion $\mathrm{m} / \mathrm{z} 307.2$ without ion $\mathrm{m} / \mathrm{z}$ 551.3 indicated that biotransformation occurred in the cyclohexene cyclic ether moiety, and metabolites with altered molecular ions with the presence of both ions $\mathrm{m} / \mathrm{z} 307.2$ and 551.3 indicated that biotransformation occurred in the disaccharide moiety. Identical results were observed for IVM- $B_{1 b}$ metabolites, but with ions $\mathrm{m} / \mathrm{z} 293.2$ and 537.3 (Figure 3). A summary of the interpretations of M1 to $M 13$ is detailed in Table 2. The microsome reactions of pure IVM- $B_{1 a}$ and $\mathrm{IVM} \mathrm{B}_{1 \mathrm{~b}}$ substrates confirmed an identical transformation pattern of both compounds (Table S1). The relative abundance of ivermectin and metabolites, generated using specific cDNA-expressed human cytochrome P450 enzymes, is provided in the supplementary information (Table S2). 
M1

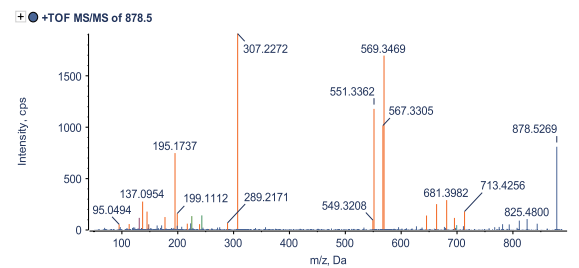

M4

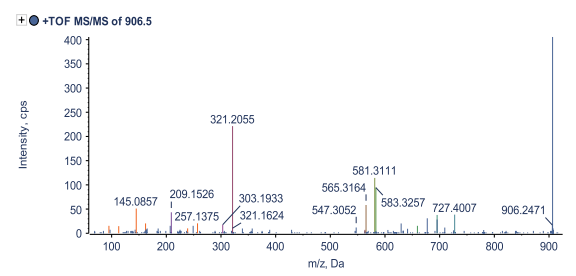

M7

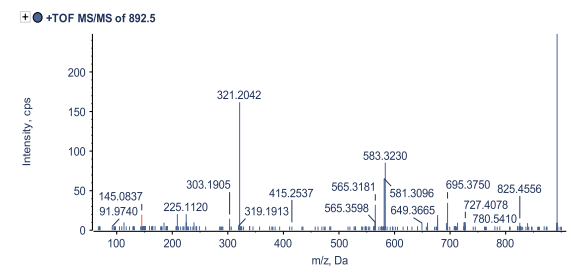

M10

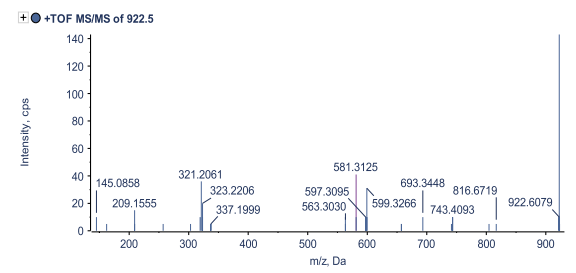

M13

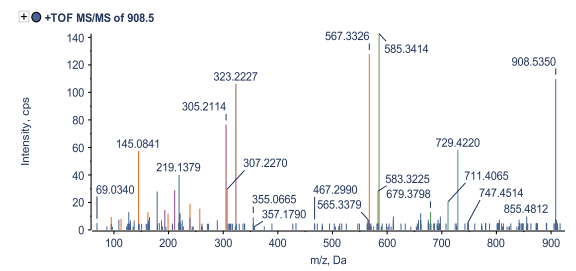

M2

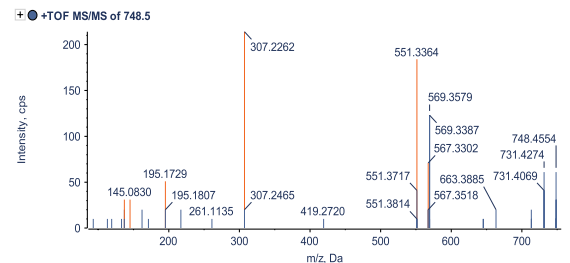

M5

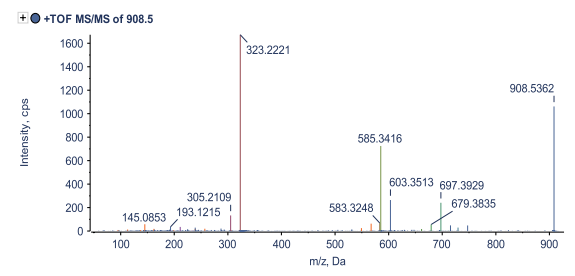

M8

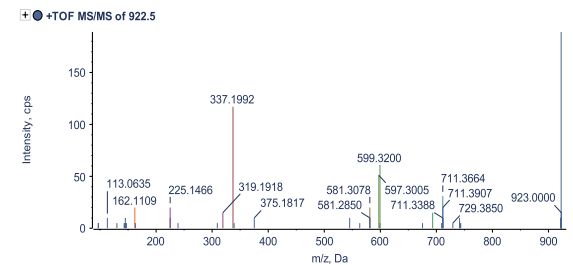

M11

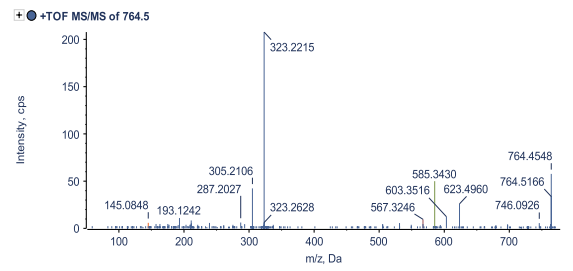

M3

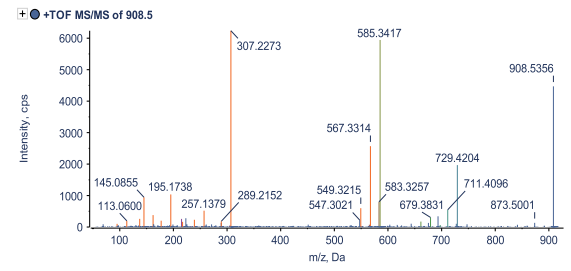

M6

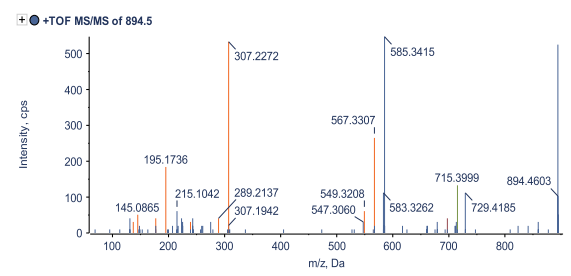

M9

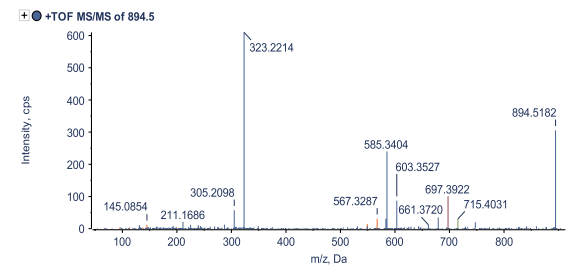

M12

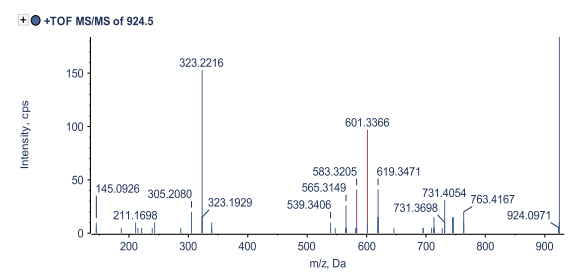

FIGURE 4 MS/MS spectra of metabolite M1 to M13. Characteristic fragment ion peaks of metabolites matched to ivermectin library peaks are shown in orange. Various characteristic neutral losses ion peaks are shown in different colors

\subsection{Confirmation of in vivo metabolite structures (M1 and M3) by LC-SPE-NMR/MS}

Microsome metabolites were separated by HPLC followed by MS and UV detection (Figure 5A) with subsequent postcolumn trapping of analytes on the SPE cartridges. Molecular formulas of the metabolites were obtained from high-resolution mass spectrometry during a preparation run. The final isolation of the metabolites was based on UV response in order to reduce the risk of contamination of the ion source because of the large amount of sample injected on the column. M1 and M3 were separated by HPLC and detected by $\mathrm{MS}$ as sodium adducts $[\mathrm{M}+\mathrm{Na}]^{+}$ions (i.e., $\mathrm{m} / \mathrm{z} 883$ and $\mathrm{m} / \mathrm{z} 913$, respectively) (Figure $5 \mathrm{~B}$ ). The NMR spectra confirmed that the demethylation of M1 occurred at C3" (labeled in yellow, Figure 6A) and that the hydroxylation of M3 occurred at C4 (labeled in yellow, Figure 6B). The evaluation of HSQC and HMBC spectra of IVM, M1, and $\mathrm{M} 3$ shows the location of biotransformation (Table 3, Figure 6, Supplement Appendix S2). Chemical shift values for proton and carbon resonances are shown in Table 3.

As the sensitivity of the $500 \mathrm{MHz}$ instrument was not sufficient to obtain an $\mathrm{HMBC}$ spectrum within the period of the nitrogen refill cycle (one week), the isolated metabolites were analyzed using an $800 \mathrm{MHz}$ instrument. This provided a complete set of NMR data (within 72 h) for structure elucidation. The HMBC correlations for IVM, M1, and M3 together with the proton spectrum obtained at $800 \mathrm{MHz}$ are detailed in the NMR supplement report. 


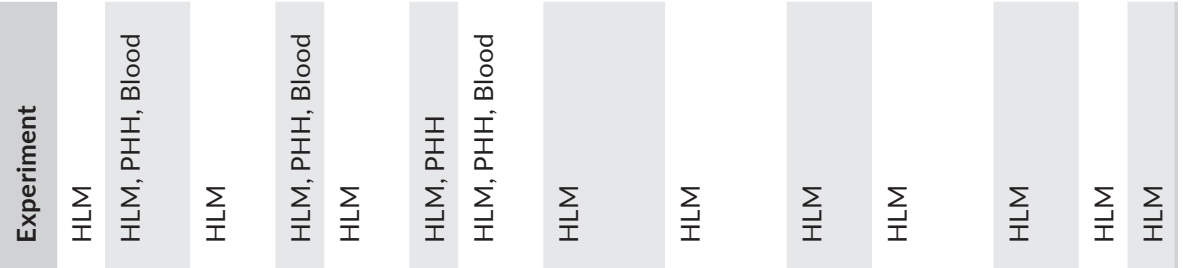

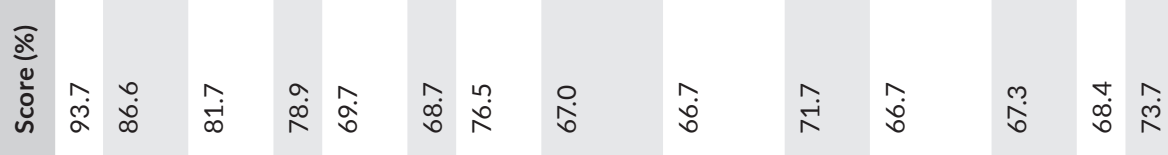

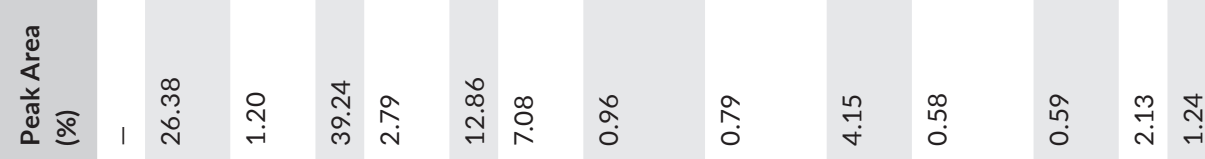

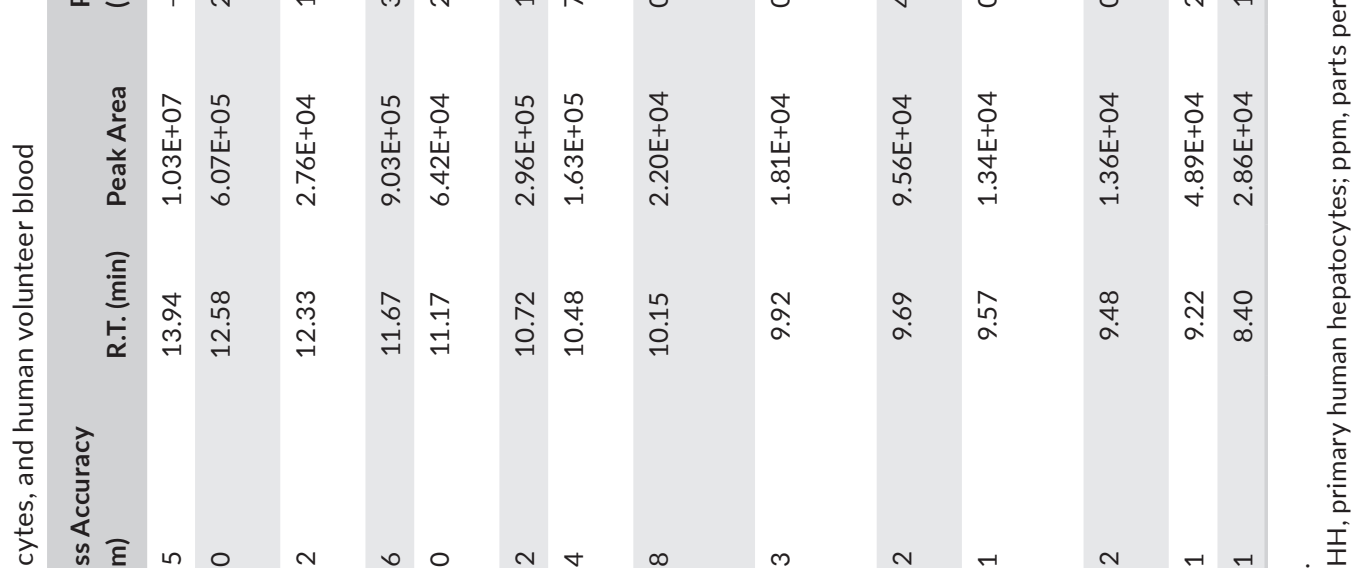

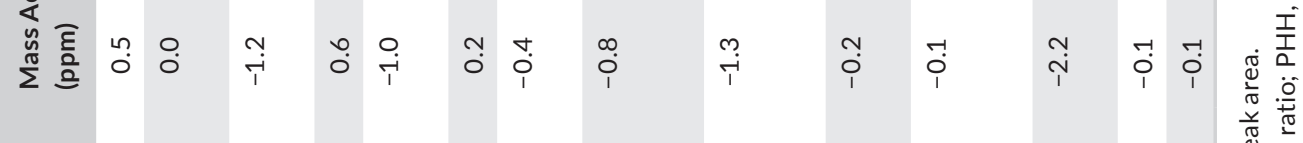

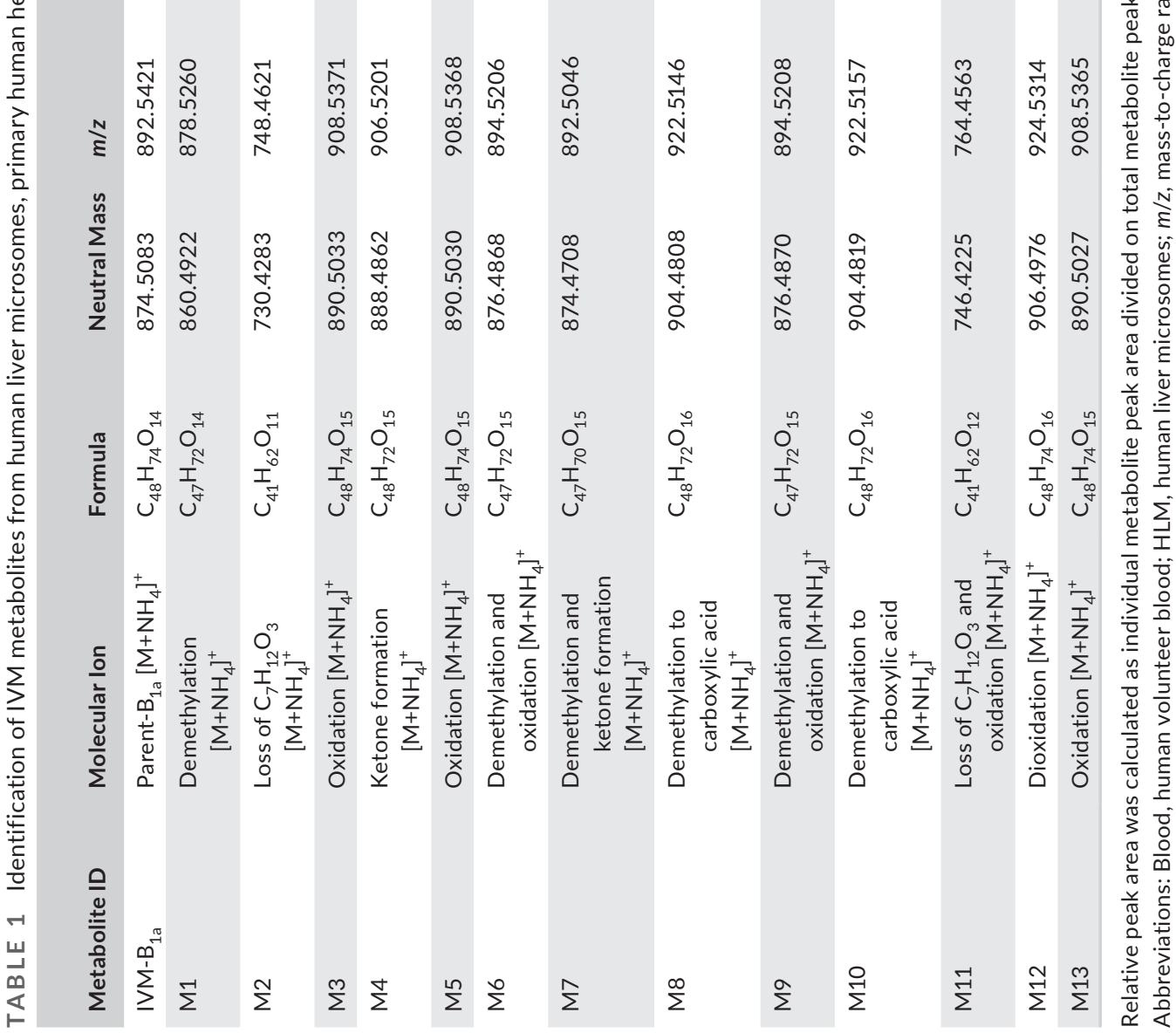


TAB LE 2 Molecular structures of metabolites predicted by accurate mass and mass fragmentation patterns

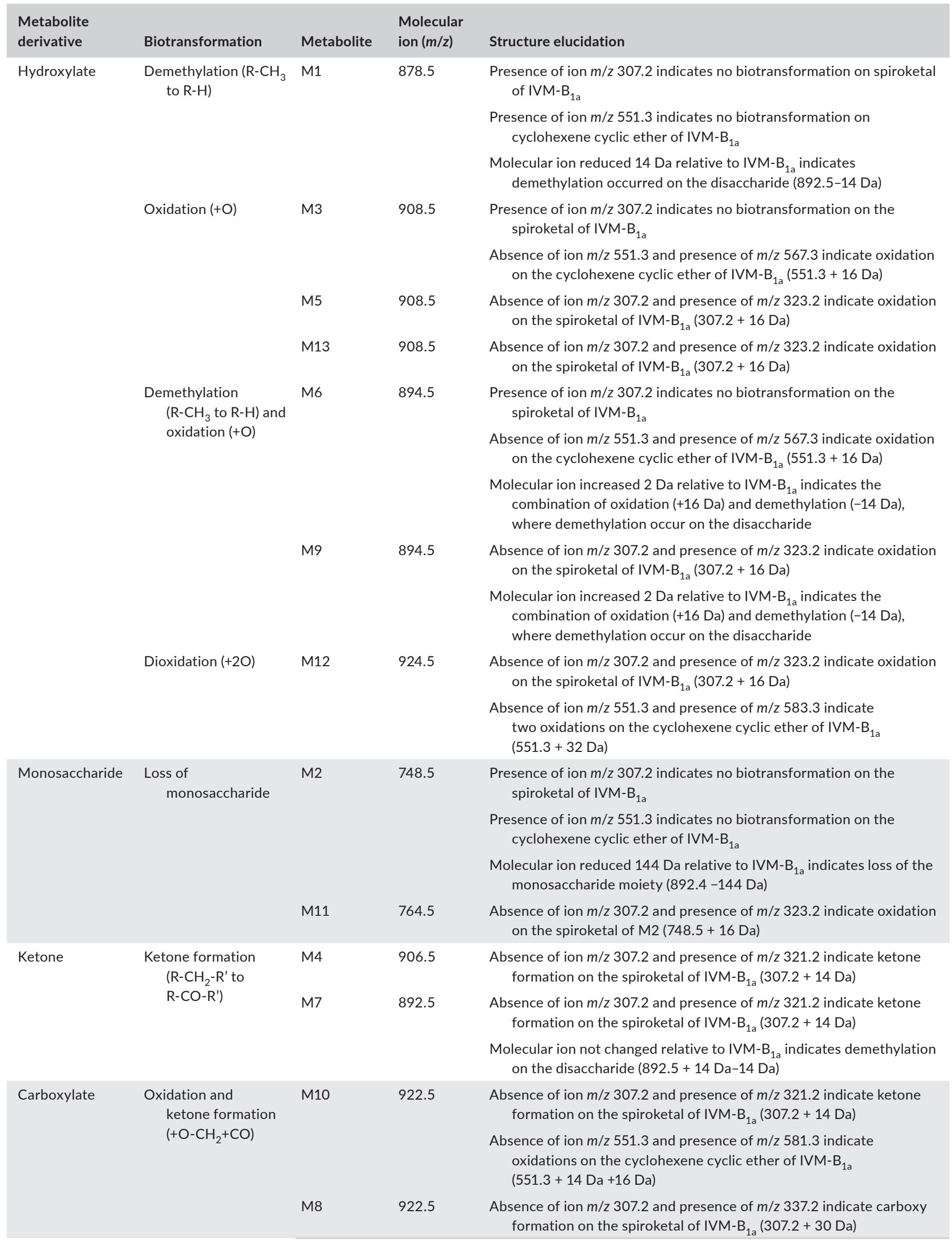


(A)

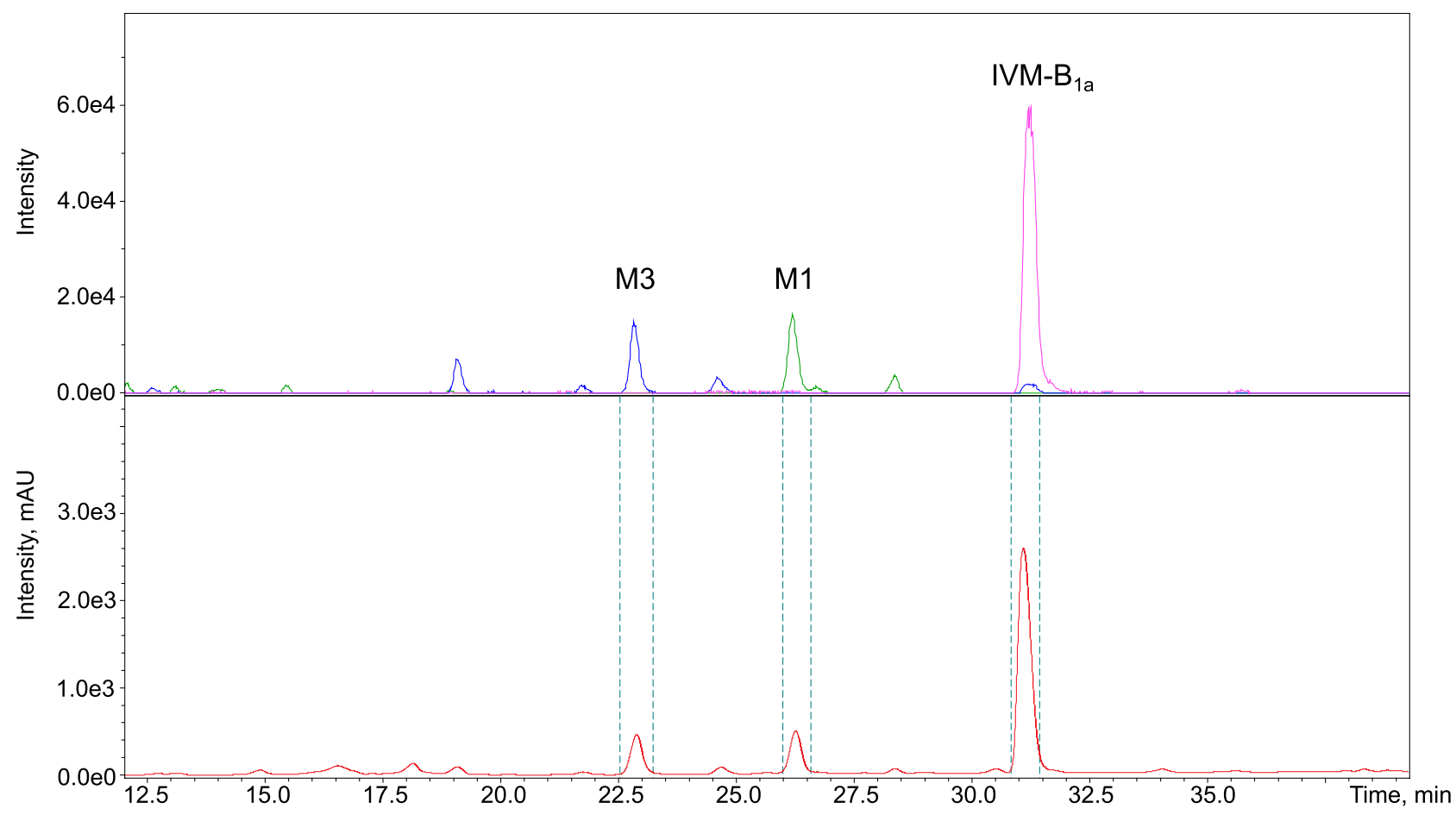

(B)

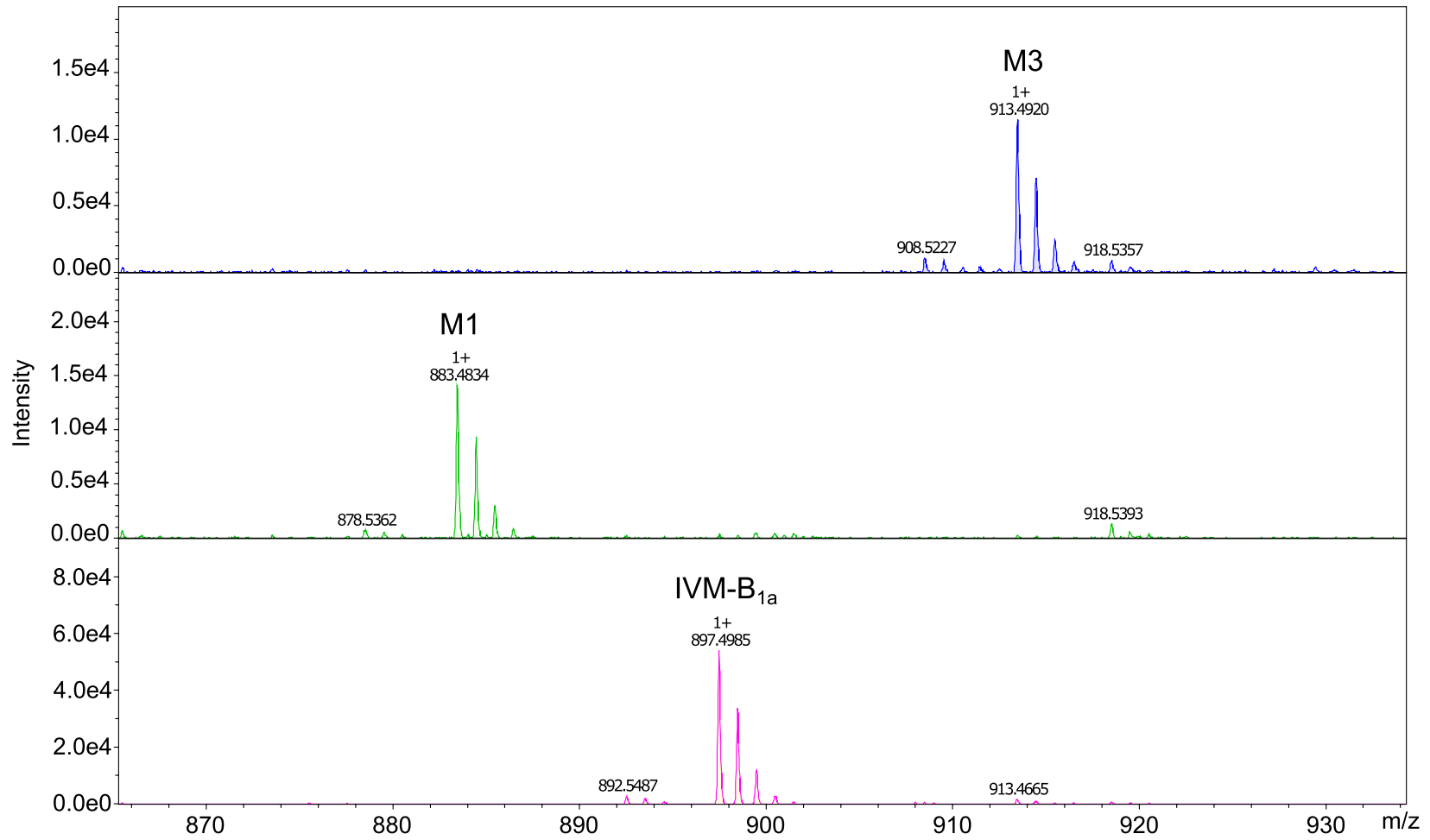

FIGURE 5 Metabolite purification and detection for NMR analysis. Panel (A) is an extracted ion chromatogram of IVM, M1, and M3 during the preparation chromatography, and UV chromatogram at $240 \mathrm{~nm}$ for the trapping experiment (the dotted lines indicate the start and end of the trapping procedure, $(B)$ is a mass spectrum from the peaks in the extracted ion chromatogram demonstrating that the molecules form stable sodium adducts 
(A)

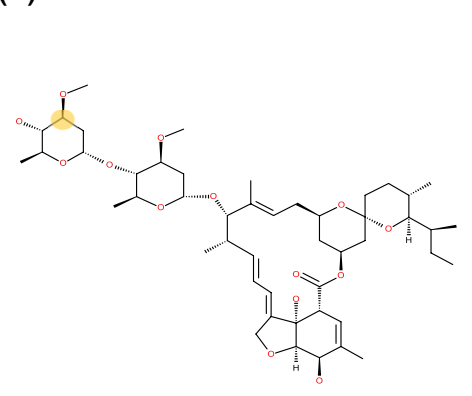

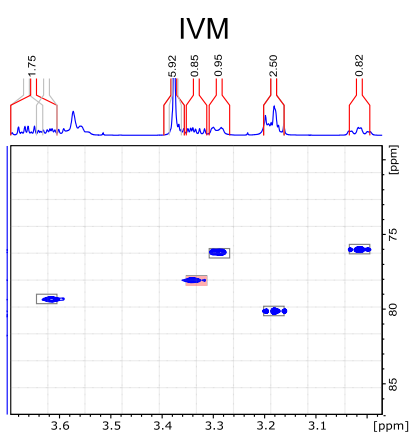



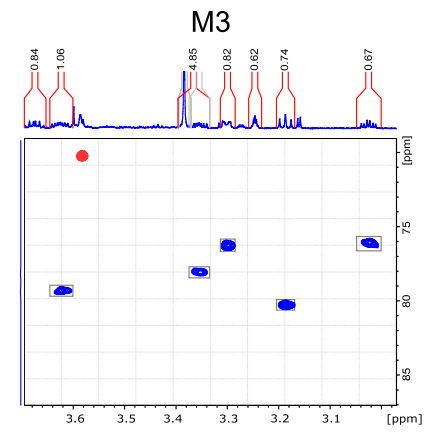

(B)

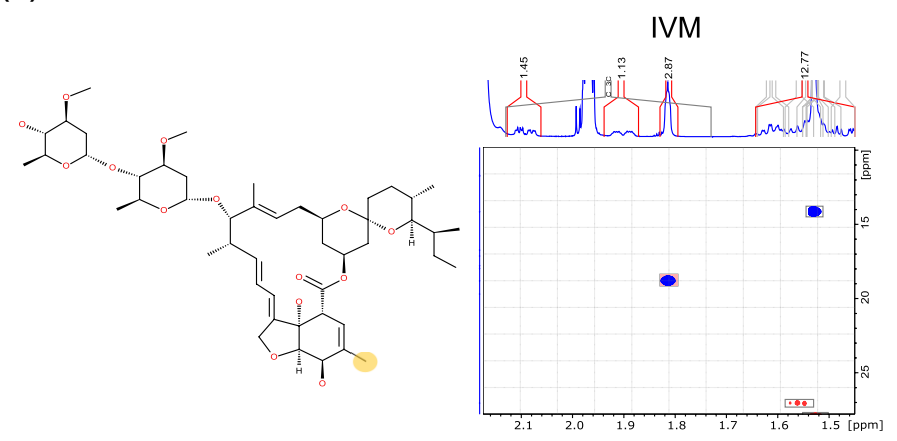

M1

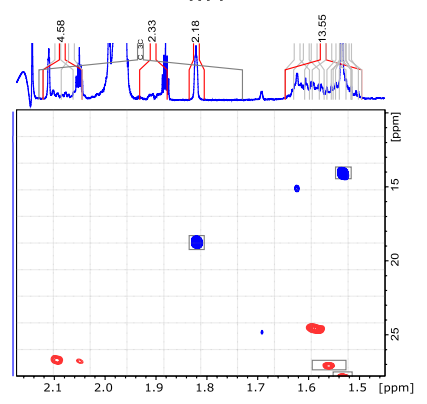

M3

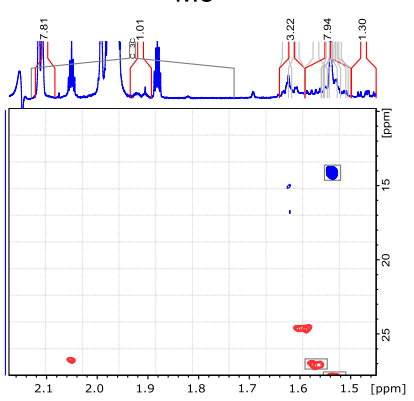

HSQC (+) HSQC (-)

FIGURE 6 NMR spectra of ivermectin (left) compared with M1 (middle) and M3 (right). The structure of IVM was labeled in yellow at signals showing the most significant differences in the spectra of the metabolites. The cursor in HSQC spectrum indicates signals missing due to changes in the metabolites. Panel (A) the shift of the $-\mathrm{CH}$ group in the metabolite $\mathrm{M} 1(\mathrm{~m} / \mathrm{z} 883)$ at the position labeled in yellow and (B) the missing $-\mathrm{CH}_{3}$ group of the metabolite $\mathrm{M} 3(\mathrm{~m} / \mathrm{z}$ 913) at position labeled in yellow. The changes in chemical shift values are listed in Table 3

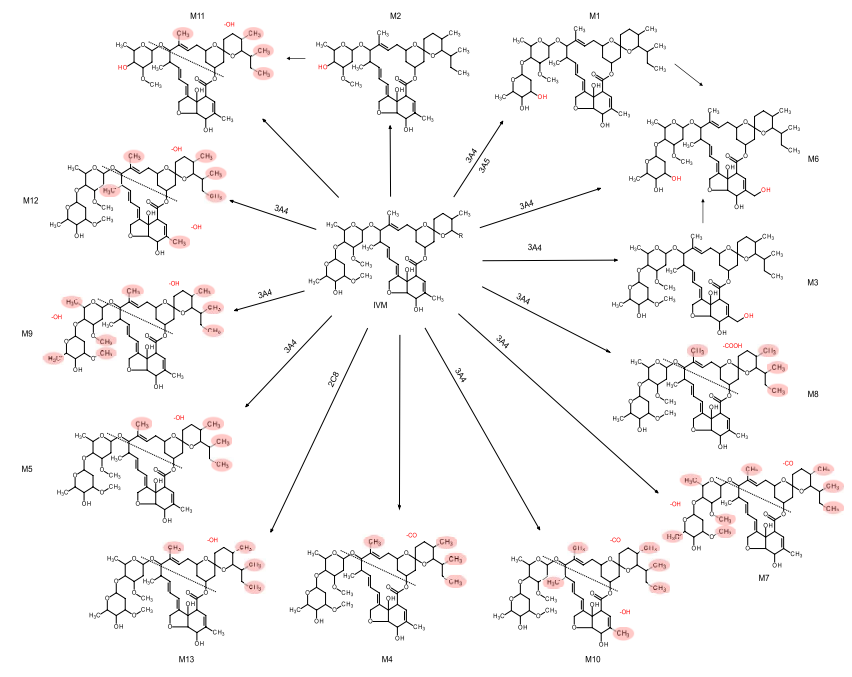

FIGURE 7 Proposed metabolic pathway of ivermectin metabolites. The exact site of biotransformation could not be confirmed for some metabolites. In these, the potential biotransformation sites are highlighted in red shading

\section{4 | Metabolic pathway characterized by pure human CYP enzyme}

IVM metabolites M1, M3, M5, M6, M7, M8, M9, M10, and M12 were all found after 60 min of incubation with CYP3A4. IVM metabolite
M1 was also produced by CYP3A5. M13 was produced by CYP2C8. None of the other investigated enzymes produced detectable amounts of the identified IVM metabolites. Proposed metabolite pathways are shown in Figure 7.

\section{4 | DISCUSSION}

The peak area of metabolites, relative to IVM, was used to estimate the relative abundance of each metabolite. In 60-min microsomes reactions, the five most abundant metabolites were M3 > M1 > M5 > M6 > M9 (Table 1, Figure 2). The four most abundant of these metabolites were also present in IVM-exposed primary human hepatocytes (M1 > M3 > M5 > M6). Three of these metabolites were found also in human volunteer blood samples taken $24 \mathrm{~h}$ after IVM administration (M1 > M3 > M6). This is the first report of IVM metabolites identified from human hepatocytes and clinical blood samples.

The IVM demethylation, oxidation, and monosaccharide metabolites identified from microsomes in this study were consistent with those reported previously from human microsomes. ${ }^{18}$ However, four additional IVM metabolites, including ketone and carboxylic derivatives, were also found in our study. Advancements in UHPLC technology 22 and state-of-the-art high-resolution mass spectrometry ${ }^{23-25}$ used here improved the sensitivity for 
TABLE 3 Chemical shift values for proton and carbon resonances obtained from HSQC and HMBC spectra of ivermectin, M1 and M3

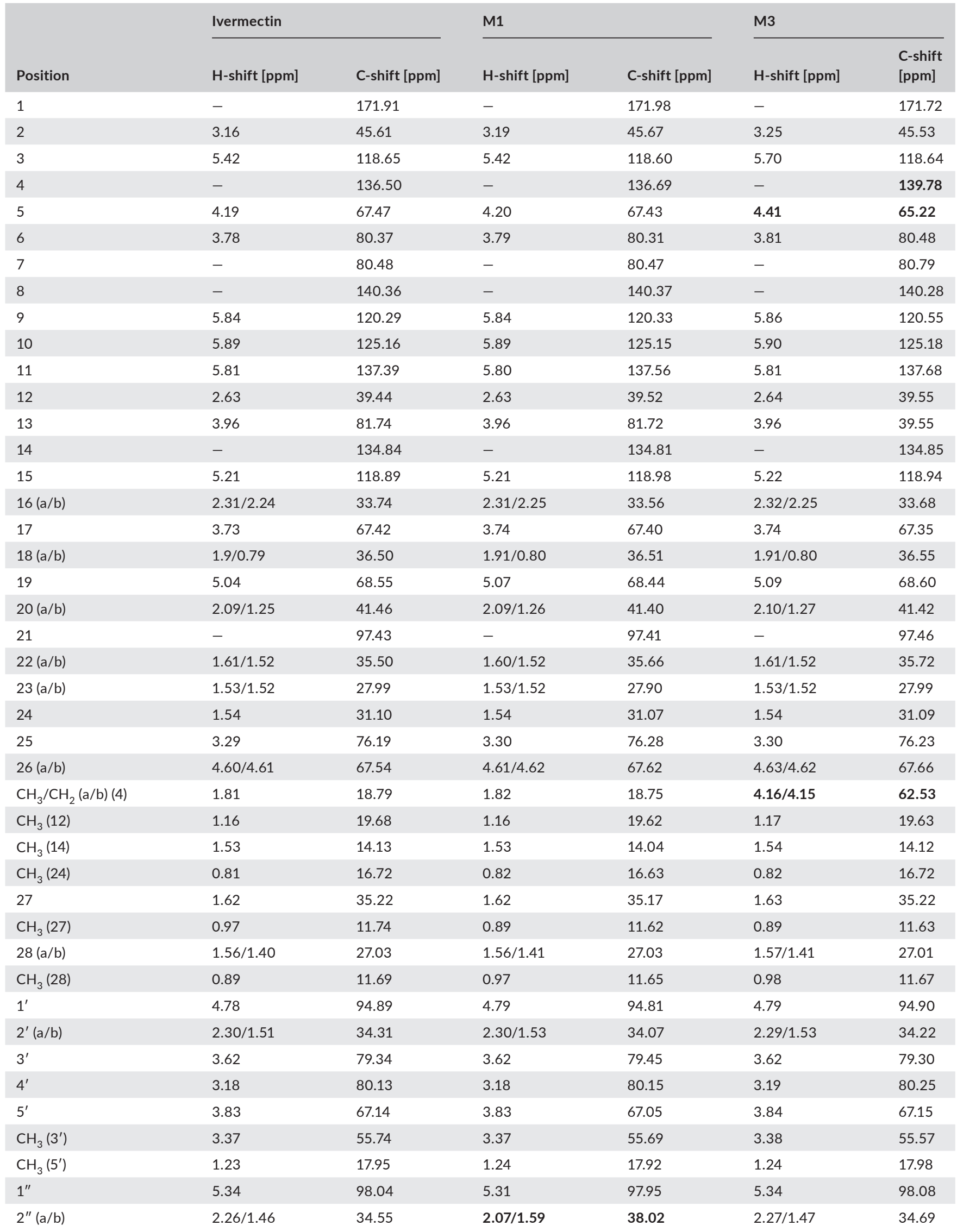


TABLE 3 (Continued)

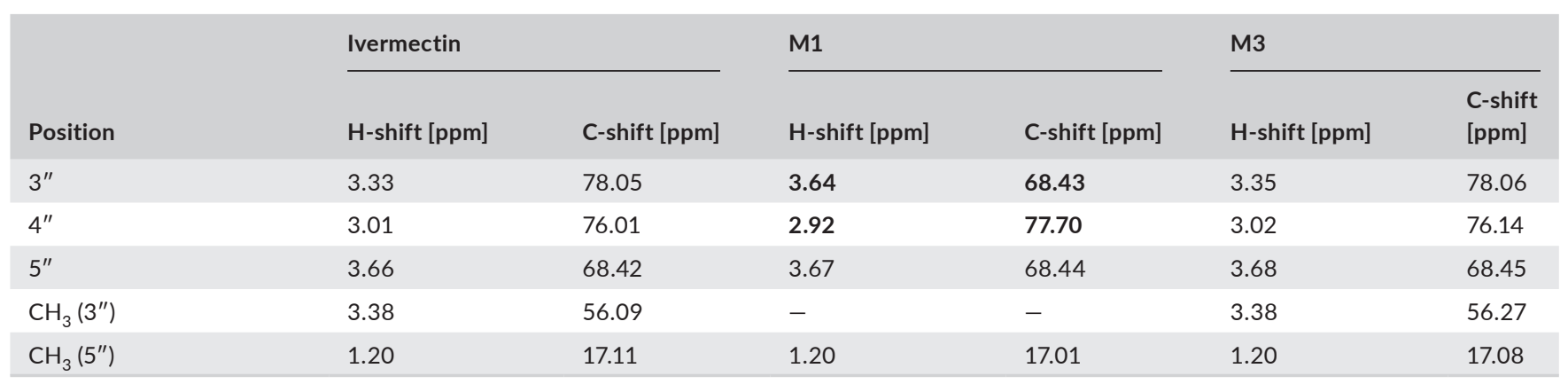

For labeling of positions refer to Figure 1. Bold fonts indicate places in the molecule where changes have occured.

metabolite detection. By using pure CYP enzymes, we showed that IVM is primarily metabolized by CYP3A4. However, we identified two additional CYP metabolism pathways of IVM; the demethylated IVM metabolite (M1) was also produced by CYP3A5 and an additional hydroxylated IVM metabolite (M13) was produced by CYP2C8. Metabolite 13 might be considered a minor metabolism pathway since it contributed $<1.5 \%$ to total metabolite peak area when ivermectin was incubated for 60 min with pooled human liver microsomes. However, it is possible that this metabolism pathway could be relatively more important in patients with functional polymorphisms in the CYP3A4 and/or CYP3A5 genes, resulting in reduced activity of CYP3A4/5 enzymes. All major metabolites identified by microsome and hepatocyte incubations, and in clinical samples were produced by CYP3A4. Metabolite 1 , which was the most abundant metabolite in hepatocyte incubations and in clinical samples, was produced both by CYP3A4 and CYP3A5. However, data presented here cannot be used to elucidate the relative in vivo contribution of each of these isoenzymes. Thus, it is likely that functional polymorphisms in CYP3A4 and/or CYP3A5 genes could be of clinical importance, since it might affect the pharmacokinetic properties of ivermectin and its metabolites. Indeed, polymorphisms in the multidrug resistance (MDR1) gene, coding for $p$-glycoprotein, and in the CYP3A gene have been linked to altered treatment response of IVM when used in onchocerciasis in a Ghanaian population. ${ }^{26}$ This warrants further pharmacogenomic evaluations in larger patient populations to elucidate the risk of treatment failures and/or adverse events in patients with functional polymorphisms.

The exact chemical structures of the two most abundant in vivo metabolites (M1 and M3) were obtained by NMR, but we could not generate enough material for NMR evaluation of the third in vivo metabolite (M6). The metabolic pathway data suggest that M1, M3, and M6 were all produced by CYP3A4, and that M6 is a combination of the demethylation and oxidation seen in $M 1$ and $M 3$, respectively. Thus, we propose that M6 is 3"-O-demethyl, 4-hydroxymethyl-ivermectin, a further (common) metabolite product of $M 1$ and $M 3$. With two sites of transformation occurring in M6 (demethylation and oxidation), it is more polar and elutes earlier than M1 and M3. Additional reversed-phase chromatography data support the M6 structure based on the elution order. The elution order in this study is also consistent with the study of Zeng et al.. ${ }^{18}$
Interestingly, many low abundance metabolites produced in microsomes were not detected from primary human hepatocytes in culture or from human volunteer blood after IVM administration. Several factors could influence the metabolic function of hepatocytes in vitro, such as initial cell suspension, confluence density of adherent cells, and drug concentration. The lower number of metabolites found in volunteer blood samples compared to microsomes could be because of phospholipids in blood samples. In vitro systems are also more efficient in producing metabolites and do not have elimination pathways (such as renal elimination) compared to in vivo systems, which could have an impact on detection results. We suggest future studies to characterize IVM metabolites produced at later time points from both hepatocytes and human blood, when metabolite abundance may possibly be altered compared to the 24-h time point thus allowing characterization of in vivo metabolism over time. To support these efforts, we have performed a clinical trial (NCT03690453) to assess the pharmacokinetic profiles of key IVM metabolites in orally treated volunteers over several weeks, and evaluated their potential mosquito-lethal and antimalarial effects. A better understanding of IVM metabolite pharmacokinetics can provide further insight into pharmacodynamics and efficacy for NTDs, especially those requiring multiple administrations such as scabies and strongyloidiasis. Furthermore, these IVM metabolites may inhibit viral replication and should be evaluated against SARS-CoV-2, the causative agent of COVID-19.

\section{5 | CONCLUSION}

We report for the first time, novel IVM metabolites from human liver microsomes, primary human hepatocytes, and from human blood after oral IVM dosing. Importantly, we identified and confirmed the structure of the two major IVM metabolites in humans; 3 "-Odemethylation IVM (M1) and 4-hydroxymethyl IVM (M3).

\section{ACKNOWLEDGMENTS}

The authors thank Markus Winterberg and Siribha Apinan for their contribution to the MS experiments. They are grateful to the volunteers for their participation in the trial, the nurses of the Clinical Therapeutics Unit and the Mahidol University Hospital for Tropical 
Diseases for their support conducting the trial. This study was funded through a grant from the Bill \& Melinda Gates Foundation (INV006052 and OPP1180249). The hepatocyte work was funded by the National Institutes of Health (R21Al149730). The Mahidol Oxford Tropical Medicine Research Unit is funded by the Wellcome Trust of Great Britain (104926/Z/14/Z, 106698/B/14/Z, 106698/Z/14/Z).

\section{CONFLICT OF INTEREST}

The authors declare no conflict of interest. We did not purchase any of the compounds or instruments mentioned in this article from Bruker.

\section{AUTHOR'S CONTRIBUTIONS}

P.T., K.C.K., and J.T. designed the research. P.T. performed MS experiments. P.T. analyzed data and wrote the first draft of the manuscript. M.G. performed NMR experiments. P.J. and B.H. performed the clinical trial. A.R. and J.H.A. performed the hepatocytes experiments. All the authors contributed to the organization of the study and the final draft of the manuscript.

\section{ETHICAL STATEMENT}

Venous blood was collected from three healthy Thai volunteers given a single dose of IVM $(400 \mu \mathrm{g} / \mathrm{kg})$. The trial was conducted at the Hospital of Tropical Medicine, Faculty of Tropical Medicine, Mahidol University in Bangkok, Thailand. The study protocol was approved by the ethics committees of the Faculty of Tropical Medicine, Mahidol University (reference number TMEC 15-004, approval number MUTM 2015-016-02), by the Oxford University Tropical Research Ethics Committee (OXTREC 4-15), and the Walter Reed Army Institute of Research (WRAIR\#2228). The trial was registered at ClinicalTrials.gov number NCT02568098. Each volunteer was provided with an explanation of the study and signed a written informed consent before study entry.

\section{DATA AVAILABILITY STATEMENT}

The data that support the findings of this study are available from the corresponding author upon reasonable request.

\section{ORCID}

Phornpimon Tipthara (D) https://orcid.org/0000-0002-4691-7063 Kevin C. Kobylinski (D) https://orcid.org/0000-0002-4807-9681 Borimas Hanboonkunupakarn (D) https://orcid. org/0000-0001-8729-1442

Alison Roth (D) https://orcid.org/0000-0001-6917-3485

John H. Adams (D) https://orcid.org/0000-0003-3707-7979

Nicholas J. White (D) https://orcid.org/0000-0002-1897-1978

Podjanee Jittamala (D) https://orcid.org/0000-0002-1020-2760

Nicholas P. J. Day (D) https://orcid.org/0000-0003-2309-1171

Joel Tarning (D) https://orcid.org/0000-0003-4566-4030

\section{REFERENCES}

1. Gonzalez P, Gonzalez FA, Ueno K. Ivermectin in human medicine, an overview of the current status of its clinical applications. Curr Pharm Biotechnol. 2012;13(6):1103-1109.
2. Caly L, Druce JD, Catton MG, Jans DA, Wagstaff KM. The FDAapproved Drug Ivermectin inhibits the replication of SARS-CoV-2 in vitro. Antiviral Res. 2020;178:104787.

3. Chaccour CJ, Kobylinski KC, Bassat Q, et al. Ivermectin to reduce malaria transmission: a research agenda for a promising new tool for elimination. Malar J. 2013;12:153.

4. Smit MR, Ochomo EO, Aljayyoussi G, et al. Safety and mosquitocidal efficacy of high-dose ivermectin when co-administered with dihydroartemisinin-piperaquine in Kenyan adults with uncomplicated malaria (IVERMAL): a randomised, double-blind, placebo-controlled trial. Lancet Infect Dis. 2018;18(6):615-626.

5. Kobylinski KC, Jittamala P, Hanboonkunupakarn B, et al. Safety, pharmacokinetics, and mosquito-lethal effects of ivermectin in combination with dihydroartemisinin-piperaquine and primaquine in healthy adult Thai subjects. Clin Pharmacol Ther. 2020;107(5):1221-1230.

6. Kobylinski KC, Foy BD, Richardson JH. Ivermectin inhibits the sporogony of Plasmodium falciparum in Anopheles gambiae. Malar J. 2012;11:381.

7. Pinilla YT, Lopes SCP, Sampaio VS, et al. Promising approach to reducing Malaria transmission by ivermectin: Sporontocidal effect against Plasmodium vivax in the South American vectors Anopheles aquasalis and Anopheles darlingi. PLoS Negl Trop Dis. 2018;12(2):e0006221.

8. Kobylinski KC, Ubalee R, Ponlawat A, et al. Ivermectin susceptibility and sporontocidal effect in Greater Mekong Subregion Anopheles. Malar J. 2017;16(1):280.

9. Alout $\mathrm{H}$, Krajacich BJ, Meyers Jl, et al. Evaluation of ivermectin mass drug administration for malaria transmission control across different West African environments. Malar J. 2014;13:417.

10. Foy BD, Alout H, Seaman JA, et al. Efficacy and risk of harms of repeat ivermectin mass drug administrations for control of malaria (RIMDAMAL): a cluster-randomised trial. Lancet. 2019;393(10180):1517-1526.

11. Campbell WC. Ivermectin and Abamectin, 1st edn. New York: Springer-Verlag; 1989.

12. Campbell WC. Ivermectin: an update. Parasitol Today. 1985; 1(1):10-16.

13. Miwa GT, Walsh JS, VandenHeuvel WJ, et al. The metabolism of avermectins B1a, H2B1a, and H2B1b by liver microsomes. Drug Metab Dispos. 1982;10(3):268-274.

14. Chiu SH, Sestokas E, Taub R, Smith JL, Arison B, Lu AY. The metabolism of avermectin-H2B1a and -H2B1b by pig liver microsomes. Drug Metab Dispos. 1984;12(4):464-469.

15. Chiu SH, Sestokas E, Taub R, et al. Metabolic disposition of ivermectin in tissues of cattle, sheep, and rats. Drug Metab Dispos. 1986;14(5):590-600.

16. Chiu SH, Carlin JR, Taub R, et al. Comparative metabolic disposition of ivermectin in fat tissues of cattle, sheep, and rats. Drug Metab Dispos. 1988;16(5):728-736.

17. Chiu SH, Taub R, Sestokas E, Lu AY, Jacob TA. Comparative in vivo and in vitro metabolism of ivermectin in steers, sheep, swine, and rat. Drug Metab Rev. 1987;18(2-3):289-302.

18. Zeng Z, Andrew NW, Arison BH, Luffer-Atlas D, Wang RW. Identification of cytochrome P4503A4 as the major enzyme responsible for the metabolism of ivermectin by human liver microsomes. Xenobiotica. 1998;28(3):313-321.

19. Roth A, Maher SP, Conway AJ, et al. A comprehensive model for assessment of liver stage therapies targeting Plasmodium vivax and Plasmodium falciparum. Nat Commun. 2018;9(1):1837.

20. Sleno $L$. The use of mass defect in modern mass spectrometry. $J$ Mass Spectrom. 2012;47(2):226-236.

21. Zhang H, Zhang D, Ray K, Zhu M. Mass defect filter technique and its applications to drug metabolite identification by high-resolution mass spectrometry. J Mass Spectrom. 2009;44(7):999-1016.

22. Churchwell MI, Twaddle NC, Meeker LR, Doerge DR. Improving LC-MS sensitivity through increases in 
chromatographic performance: comparisons of UPLC-ES/MS/MS to HPLC-ES/MS/MS. J Chromatogr B Analyt Technol Biomed Life Sci. 2005;825(2):134-143.

23. Theodoridis GA, Gika HG, Want EJ, Wilson ID. Liquid chromatography-mass spectrometry based global metabolite profiling: a review. Anal Chim Acta. 2012;711:7-16.

24. Meyer MR, Maurer HH. Current applications of high-resolution mass spectrometry in drug metabolism studies. Anal Bioanal Chem. 2012;403(5):1221-1231.

25. Ramanathan R, Jemal M, Ramagiri S, et al. It is time for a paradigm shift in drug discovery bioanalysis: from SRM to HRMS. J Mass Spectrom. 2011;46(6):595-601.

26. Kudzi W, Dodoo AN, Mills JJ. Genetic polymorphisms in MDR1, CYP3A4 and CYP3A5 genes in a Ghanaian population: a plausible explanation for altered metabolism of ivermectin in humans? BMC Med Genet. 2010;11:111.

\section{SUPPORTING INFORMATION}

Additional supporting information may be found online in the Supporting Information section.

How to cite this article: Tipthara P, Kobylinski KC, Godejohann M, et al. Identification of the metabolites of ivermectin in humans. Pharmacol Res Perspect.

2021;9:e00712. https://doi.org/10.1002/prp2.712 